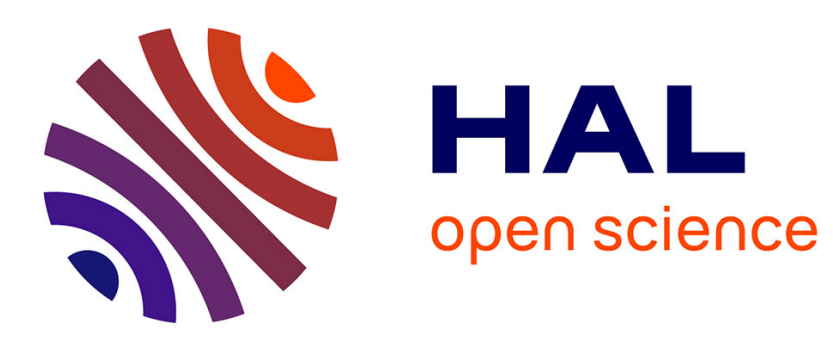

\title{
Sensitive detection of multiple thresholds for electric field ionization of sodium rydberg states
}

\author{
J.-L. Vialle
}

\section{To cite this version:}

J.-L. Vialle. Sensitive detection of multiple thresholds for electric field ionization of sodium rydberg states. Journal de Physique Lettres, 1978, 39 (21), pp.365-368. 10.1051/jphyslet:019780039021036500 . jpa-00231518

\section{HAL Id: jpa-00231518 https://hal.science/jpa-00231518}

Submitted on 1 Jan 1978

HAL is a multi-disciplinary open access archive for the deposit and dissemination of scientific research documents, whether they are published or not. The documents may come from teaching and research institutions in France or abroad, or from public or private research centers.
L'archive ouverte pluridisciplinaire HAL, est destinée au dépôt et à la diffusion de documents scientifiques de niveau recherche, publiés ou non, émanant des établissements d'enseignement et de recherche français ou étrangers, des laboratoires publics ou privés. 


\title{
SENSITIVE DETECTION OF MULTIPLE THRESHOLDS FOR ELECTRIC FIELD IONIZATION OF SODIUM RYDBERG STATES
}

\author{
J.-L. VIALLE \\ Laboratoire Aimé-Cotton, C.N.R.S. II, Bât. 505, 91405 Orsay, France
}

(Reçu le 5 juin 1978, accepté le 15 septembre 1978)

\begin{abstract}
Résumé. - On présente, dans cette Lettre, une nouvelle méthode permettant d'étudier l'ionisation par un champ électrique statique d'atomes dans des états hautement excités (états de Rydberg). Cette méthode est particulièrement bien adaptée au cas où un niveau atomique donné présente plusieurs seuils d'ionisation. Une expérience effectuée sur l'état $20 \mathrm{~d}$ du sodium illustre les avantages de cette méthode.
\end{abstract}

\begin{abstract}
This paper presents a new method for studying the ionization of highly excited atomic Rydberg states by a static electric field. This method is especially well adapted when several ionization thresholds appear for a given atomic level. The advantages of the method are illustrated on the $20 \mathrm{~d}$ state of $\mathrm{Na}$.
\end{abstract}

1. Introduction. - The development of dye lasers tunable over a wide frequency range, allows the selective excitation of highly excited levels in atoms [1-4]. The most efficient method to detect atoms in such highly excited levels is based on field ionization [5]. Moreover, field ionization is not only a suitable detection technique but also a very interesting physical phenomenon in itself [6]. The ionization threshold for a given state is expected to be sharply defined because the field ionization rate must vary very rapidly with the ionizing field value. This has been effectively observed by Ducas et al. [7] for the $n$ s levels of $\mathrm{Na}$. However, on the contrary they have observed wider thresholds for the $n$ d levels and suggested that this effect was due to the presence of several adjoining thresholds because of various $\left|m_{L}\right|$ states in a d level. Gallagher et al. $[8,9]$ have generally observed two and three thresholds for, respectively, $n \mathrm{p}$ and $n \mathrm{~d}$ levels in sodium. More surprisingly, they also observed several thresholds for some non-degenerate levels ( $n \mathrm{~d},\left|m_{L}\right|=2$ for $n \geqslant 18$ ) and attributed these multiple thresholds to partly diabatic passage from zero electric field to high ionizing field, due to the too fast rise time of the electric field. The method described in this paper is especially designed to study multiple ionization thresholds.

2. Principle of the experiment. - The sodium atoms of an atomic beam are stepwise excited by a C. W. laser and a pulsed dye laser; thus, a $n$ s or $n$ d Rydberg level can be selectively populated via the $3 p$ level. By use of suitable polarized lights it is possible to put atoms in these highly excited states, all with the same given value for $\left|m_{L}\right|$. One microsecond after the optical excitation, a pulsed high electric field is established over the interaction region so that the atoms in the Rydberg state are field ionized. The ions are sent onto an electron multiplier and counted. The classical method to study critical ionization fields is to use a pulsed electric field with a fast rise time and a nearly flat top and to measure the number of ions produced versus the maximum value of the electric field. The result is a curve presenting several plateaus; each increase of the signal between two adjacent plateau corresponds to a threshold field value. But if one applies in the interaction region a pulsed electric field whose time dependence is shown in figure 1 and if the counting system counts the ions only when the gate, also shown in figure 1 , is opened, then the ion signal is quite different. As long as the critical field $F_{\mathrm{c}}$ of the studied state is lower than $F_{0}$ (case $a$, Fig. 1) the pulses given by the multiplier are received by the counter before the gate is opened and therefore they are not counted. If the critical field $F_{\mathrm{c}}$ is greater than $F_{0}+\Delta F$, field ionization does not occur. Only in case $b$ of figure $1\left(F_{0}<F_{\mathrm{c}}<F_{0}+\Delta F\right)$ are the pulses of the multiplier received when the gate is opened and they are counted. $\Delta F$ is taken to be very small compared to $F_{0}\left(\frac{\Delta F}{F_{0}} \simeq 2 \times 10^{-2}\right)$ and 

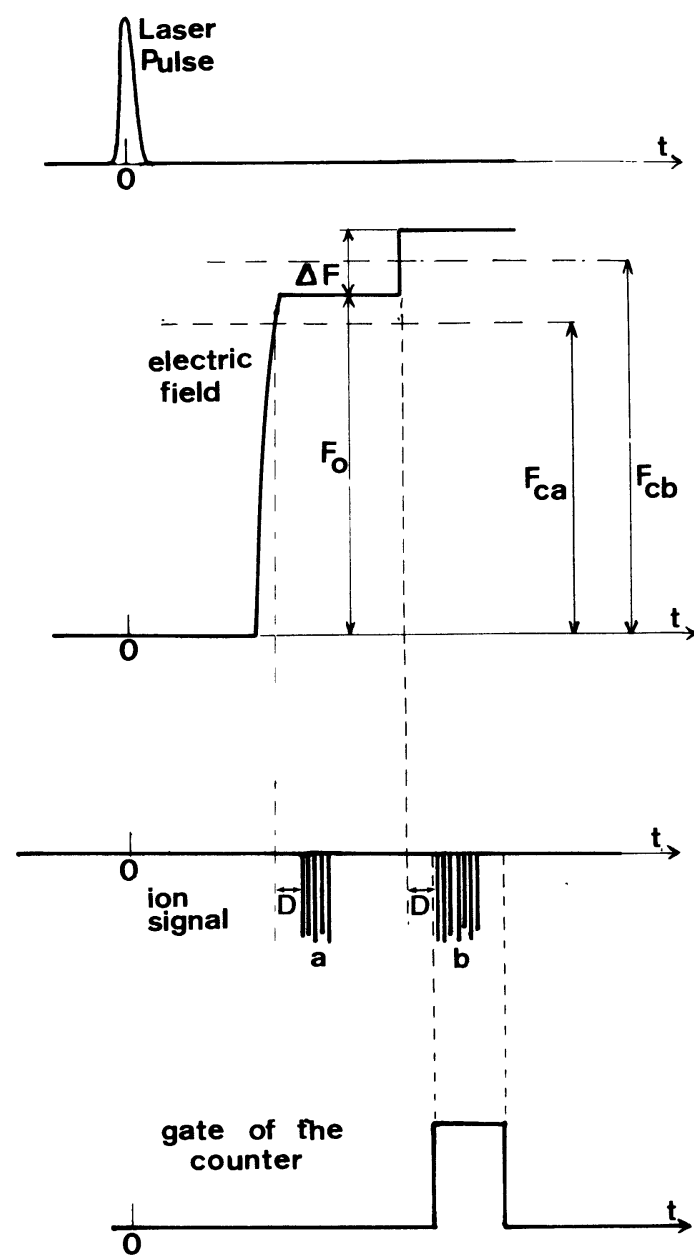

FIG. 1. - Timing of the pulsed electric field and of the counting system. The delay $D$ corresponds to the transit time of the ions from the interaction region to the detector.

it has a fixed value while $F_{0}$ is varied. The number of ions counted versus ionizing field value $F_{0}$ with this field shape is represented by a curve having a series of peaks : each peak corresponds to an ionization threshold. One can easily understand that this curve, if $\Delta F$ is sufficiently small, is approximately the derivative of the curve obtained by the classical technique.

3. Experimental set-up. - By using suitably polarized exciting lights, it is possible to get highly excited atoms with a selected $\left|m_{L}\right|$ value, $m_{L}$ being the projection, on the pulsed electric field direction, of the electron's orbital angular momentum $\mathbf{L}$. This is shown in figure 2.

The first step excitation is always carried out with a $\sigma^{+}$polarized light (frequency $v_{1}$ ), with respect to the direction of $\mathbf{F}$, allowing us to reach, by Zeeman optical pumping, an intermediate state for which $m_{L}=+1$ is a good quantum number. On the other hand the light inducing the second step excitation (frequency $v_{2}$ ) is set onto the atomic beam and it can be polarized in three different ways : $\sigma^{+}$(Fig. 2a), $\pi$ (Fig. $2 b$ ) or $\sigma^{-}$(Fig. $2 c$ ) which lead respectively to $m_{L}=+2,+1$ or 0 in the highly excited state.

The ionized atoms are accelerated by the electric field $\mathbf{F}$ and therefore the ion beam is very close and parallel to one light beam at least. Therefore the ion beam must be deflected by another electric field $\mathbf{F}^{\prime}$ in order to be sent onto the electron multiplier without screening the light beam. The interaction region of the atomic beam of sodium with the two dye lasers corresponding to the $a$ and $c$ cases of figure 2 (final states $m_{L}=+2$ or 0 ) is shown in figure 3 . The two laser beams are colinear, propagating in opposite directions and perpendicularly to the atomic beam direction. (To get the optical excitation shown in figure $2 b$ the second laser beam $\left(v_{2}\right)$ is sent to the atomic beam perpendicularly to the plane of the figure and linearly polarized parallel to F.)

The first laser, a C. W. single mode commercial dye laser, induces the transition $3{ }^{2} \mathrm{~S}_{1 / 2}, F=2 \rightarrow 3{ }^{2} \mathrm{P}_{3 / 2}$, $F^{\prime}=3$. The laser light is $\sigma^{+}$circularly polarized by a quarter wave plate $\mathrm{L}_{1}$. The fluorescence signal of the atomic beam is used to lock the frequency $v_{1}$ of the (c) (b)

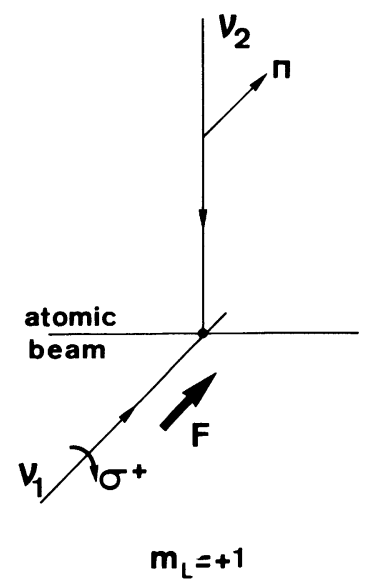

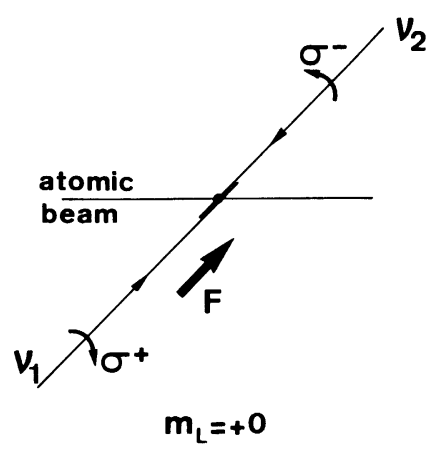

FIG. 2. - Schema of the three different possible arrangements for the two step optical excitation (light polarizations and beam directions) allowing the population of highly excited states with a selected $m_{L}$ value. Case $a: m_{L}=+2$; Case $b: m_{L}=+1 ;$ Case $c: m_{L}=0$. 


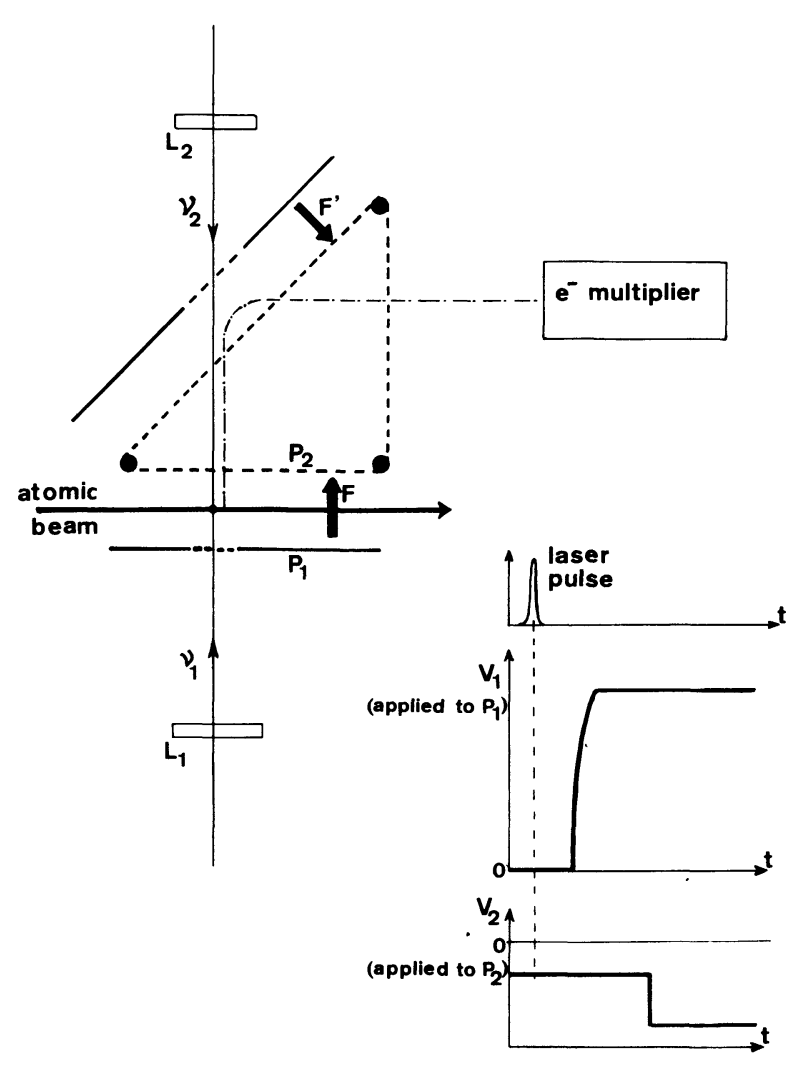

FIG. 3. - Schematic view of the interaction region with the trajectory of the ions (- - - - - - ). Right bottom : time dependence of the electric potentials of field plates $P_{1}$ and $P_{2}$. Notice that $\mathbf{F}$ (proportional to $V_{1}-V_{2}$ ) has the time dependence indicated in figure 1 .

laser light on the atomic transition frequency. The population of the $F=2$ sublevel of the ground state is initially equally distributed between all its Zeeman sublevels. By Zeeman optical pumping it is possible, after a very short interaction time, to get all these atoms only in the $F=2, m_{F}=2$ Zeeman sublevel and then only the sublevel $F^{\prime}=3, m_{F^{\prime}}=3$ of ${ }^{2} \mathrm{P}_{3 / 2}$ can be reached. For this particular sublevel $m_{L}$ is a good quantum number and remains equal to +1 .

The second laser is a pulsed tunable dye laser pumped by the red line $(\lambda=659 \mathrm{~nm})$ of a frequency doubled NdYAG laser. The infrared tunable radiation of this dye laser is converted, by frequency doubling, into violet light, which interacts with the atomic beam. The scanning range for the frequency $v_{2}$ of this second laser light allows us to populate the Rydberg $n$ s and $n$ d levels from $n=10$ up to the continuum. The pulsed dye laser light is focused on the atomic beam into a spot entirely located inside the C. W. laser beam, so that each atom which interacts with the pulsed laser is prepared in the $F^{\prime}=3$, $m_{F^{\prime}}=3$ sublevel. By use of a quarter wave plate $L_{2}$ the pulsed laser light is $\sigma^{+}$or $\sigma^{-}$polarized corresponding respectively to cases $a$ or $c$ of figure 2 .

The interaction region (Fig. 3) is located between two electric field plates $\left(P_{1}\right.$ and $\left.P_{2}\right)$. The pulsed elec- tric field $\mathbf{F}$ is applied, after laser excitation, between these two plates. Plate $P_{1}$ has a grid in its centre, allowing the passage of the laser beams and plate $P_{2}$ is one side of an equipotential prism obtained by winding a metallic wire on three parallel threaded rods. These two plates are electrically insulated from the ground, and electric potentials $V_{1}$ and $V_{2}$ can be applied to generate the ionizing field $\mathbf{F}$. The ions created and accelerated by the electric field $\mathbf{F}$ are repelled and deflected by a second electric field $\mathbf{F}^{\prime}$ and they are sent onto the electron multiplier. This arrangement works like a totally reflecting prism. With this geometry the ionizing electric field is parallel to the direction of the laser beams as required.

The electric field shape shown in figure 1 is generated in the following way : $1 \mu$ s after the laser pulse, a step-shaped high electric voltage $V_{1}$ is applied to plate $P_{1}$, and, with a larger delay ( 2 or $3 \mu \mathrm{s}$ ), a small step-shaped electric voltage $V_{2}$ is applied to plate $\mathrm{P}_{2}$. A permanent d.c. voltage can be also added to the voltage of plate $\mathrm{P}_{2}$ (see Fig. 3) the usefulness of which will be explained later.

4. Results and conclusions. - Results on the $20 \mathrm{~d}$ level are presented in figure 4. Starting from $m_{L}=+1$ for the intermediate state, $\sigma^{+}$of $\sigma^{-}$polarization for the second step excitation leads respectively to the $m_{L}=+2$ or $m_{L}=0$ sublevel. Therefore, it is possible to study separately ionization thresholds for the 20d $\left|m_{L}\right|=2$ and $20 \mathrm{~d}\left|m_{L}\right|=0$ sublevels provided that the highly excited atoms keep the $m_{L}$ value unchanged from the optical excitation time, till the electric field is switched on. For this reason, a permanent d.c. voltage is added to the voltage of the plate $\mathrm{P}_{2}$, creating a weak permanent d.c. field in the same direction as the pulsed electric field. The permanent field strength is such that the Stark effect is stronger than spin-orbit interaction and therefore $\left|m_{L}\right|$ is a good quantum number for the highly excited atoms and remains constant. On the other hand the permanent field is kept sufficiently weak so that only the $\mathrm{d}$ level is appreciably populated.

The results presented in figure 4 are obtained with a d.c. field magnitude of 3.3 . V/cm. Curves A are obtained by the classical method and by counting all produced ions, curves $B$ are obtained by the method described above. Corresponding thresholds in cases $A$ and $B$ are identically numbered.

The advantage of the present method appears clearly on the curves shown. For a level with several thresholds the higher ones are much better resolved and visible since the background due to atoms ionized at a lower field value and the associated noise are suppressed.

The 20d state is the highest state studied in ref. [9]. As one can see in table I the results obtained by Gallagher et al. are only partly confirmed by the present results. 
20d

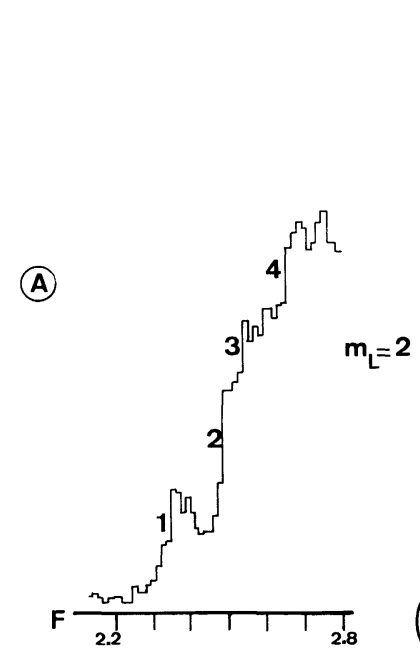

$$
F_{d c}=3.3 \mathrm{~V} / \mathrm{cm}
$$
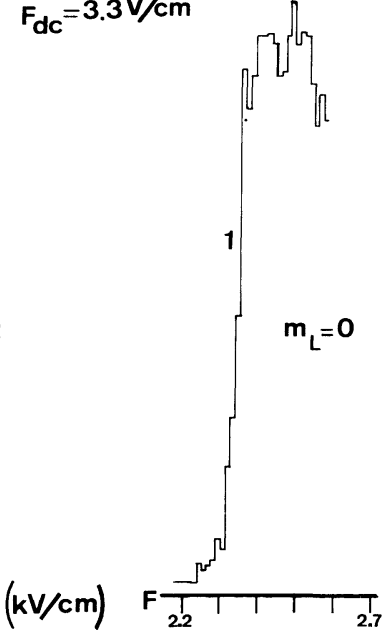

(B)

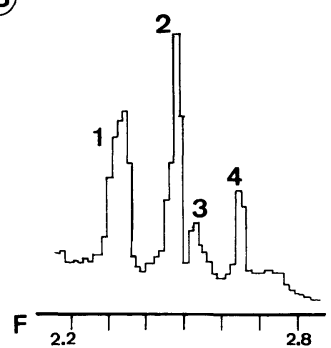

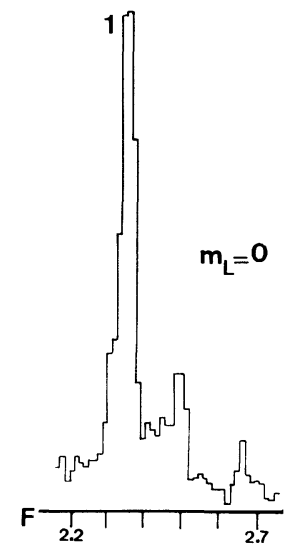

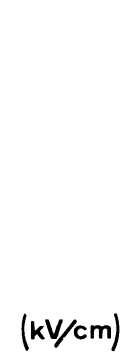

$(\mathrm{kV} / \mathrm{cm}) \mathrm{F} \frac{1.2}{2.2}+\frac{1}{2} \frac{1}{2.7}$

Fig. 4. - Ion signal as a function of the pulsed electric field $\mathbf{F}$ for the 20d state : A) By the classical method; B) By the present method. The experimental conditions for these recordings were the following : rise time of $F_{0}=1 \mu \mathrm{s}$; delay between $F_{0}$ and $\Delta F=2 \mu \mathrm{s} ;$ gate open time $=1 \mu \mathrm{s} ;$ amplitude of $\Delta F \simeq 30 \mathrm{~V} / \mathrm{cm}$; permanent d.c. field $F_{\text {d.c. }} \simeq 3.3 \mathrm{~V} / \mathrm{cm}$. At $F_{\text {d.c. }}$ field strength used the selection of a given $m_{L}$ value is not totally efficient, therefore the peaks corresponding to $m_{L}=2$ appear weakly on the right side of figure $4 \mathrm{~B}$ just as the peak corresponding to $m_{L}=0$ is seen on the left side of figure $4 \mathrm{~B}$.

TABLE I

Threshold fields $(\mathrm{kV} / \mathrm{cm})$ for the $20 \mathrm{~d}$ state of $\mathrm{Na}$

$\begin{array}{cccc} & \left|m_{L}\right|=0 & \left|m_{L}\right|=1 & \left|m_{L}\right|=2 \\ - & - & - & - \\ \text { Gallagher } & 2.22 & 2.30 & 2.48 \\ \text { et al. } & & & 2.62 \\ & & & \\ & & & 2.48 \\ \text { Present } & 2.33 & 2.33 & 2.54 \\ \text { work } & & & 2.66 \\ & & & 2.76\left(^{*}\right)\end{array}$

$\left(^{*}\right)$ The signal for this threshold is very weak.

The uncertainty in the absolute values of the measured electric field thresholds is estimated to be $4 \%$, half of which is due to the determination of the electric field value in the interaction region. Taking into account the uncertainties of the two measurements all the present results are consistent with the results of Gallagher. Moreover, only one ionization threshold is observed for the $\left|m_{L}\right|=0$ and $\left|m_{L}\right|=1$ states, whereas the $\left|m_{L}\right|=2$ state presents several thresholds confirming his observation. On the other hand, the ratio of one threshold value to another is determined with a better accuracy and the present method allows the separation of two thresholds which differ by $2 \%$ at least; nevertheless, and contrary to the results of Gallagher, the difference between the observed thresholds for $\left|m_{L}\right|=0$ and $\left|m_{L}\right|=1$ is too small to be measured.

These results will be discussed in a more detailed paper, together with other results obtained in the same way for $n \mathrm{~s}, n \mathrm{p}$ and $n \mathrm{~d}$ levels.

\section{References}

[1] Fabre, C., Gross, M. and Haroche, S., Opt. Commun. 13 (1975) 393.

[2] Stebbings, R. F., Lattimer, C. J., West, W. P., Dunning, F. B. and Cook, T. B., Phys. Rev. A 12 (1975) 1453.

[3] Ferguson, A. I. and DunN, M. H., Opt. Commun. 23 (1977) 227.

[4] Camus, P., Champeau, R.-J., Feneuille, S., Liberman, S., Morillon, C. and Pinard, J., Colloques Internationaux du C.N.R.S., No 273 : Etats atomiques et moléculaires couplés à un continuum. Atomes et molécules hautement excités. (1977) pp. 67-74, Editions du C.N.R.S., Paris.
[5] Tuan, D. H., Liberman, S. and Pinard, J., Opt. Commun. 18 (1976) 533.

[6] Littman, M. G., Zimmerman, M. L. and Kleppner, D., Phys. Rev. Lett. 37 (1976) 486.

[7] Ducas, T. W., Littman, M. G., Freeman, R. R. and KlepPNer, D., Phys. Rev. Lett. 35 (1975) 366.

[8] Gallagher, T. F., Humphrey, L. M., Hill, R. M. and Edelstein, S. A., Phys. Rev. Lett. 37 (1976) 1465.

[9] Gallagher, T. F., Humphrey, L. M., CoOKe, W. E., Hill, R. M. and Edelstein, S. A., Phys. Rev. A 16 (1977) 1098. 